\title{
Recovery of Residues from Olive Industry: Characterization of Simple and Acetylated Lignin
}

\author{
Amine Abid $^{1 *}$, Fatima Charrier El Bouhtoury ${ }^{2}$, Slimane Gabsi ${ }^{1}$ \\ ${ }^{1}$ National School of Engineering of Gabes, Energy, Water, Environment and Process Laboratory, University of Gabes, Omar Ibn \\ ElKhattab-6029 Gabes, Tunisia \\ ${ }^{2}$ Institute of Analytical Sciences and Physico-Chemistry for the Environment and Materials IPREM, University of Pau and Pays \\ l'Adour, Technopôle Helioparc 2 Avenue Pierre Angot 64053 Pau Cedex 9, France
}

Corresponding Author Email: abidamine@ hotmail.fr

https://doi.org/10.18280/acsm.440504

Received: 9 February 2020

Accepted: 20 September 2020

\section{Keywords:}

lignin organosolv, polyol, acetylation, characterization physico-chemical analyzes, sugar

\begin{abstract}
Lignin is a renewable material obtained in large quantities as a by-product of the olive industry. In this context, this study aims to examine lignin as macro-monomers in the synthesis of rigid polyurethane foam after chemical modification. Indeed, the lignin extracted from the olive residue is used by chemical treatment according to the organosolv process. Comparison by characterization of the single isolated lignin and the acetylated lignin according to the physicochemical analysisTGA, DSC, FTIR, C-13 NMR, 1-H NMR, and molar mass distribution (sec) shows its value as biomass capable of being transformed into polyol afterchemical modification. acetylation increases the weight and the number of molar masses by $25-50 \%$ was also determined. Acetylation has a greater effect on the short chains which are probably richer in free phenolic groups. Mass distribution analyzes reveal a relatively high molar mass in organosolv lignin moreover, polydispersity values are very high $(20>>1)$. The characterization of spent olive cake reflects the interest of using this biomass as a source of renewable energy for the production of industrial polymers.
\end{abstract}

\section{INTRODUCTION}

Biomass is now the second renewable energy in the world. Tunisia is one of the Mediterranean countries renowned for the production of olive oil with an average production of 180,000 tons of oil during the last five years $[1,2]$. In this work, the emphasis is placed on the recovery of waste from oil mills (olive residue) in the sense that they constitute a potential source of additional revenue likely to contribute to improving the profitability of olive-growing farms [3]. Different techniques are used to transform this olive pomace into forms of energy that can be incorporated into our lives. It is in this context that the present study is situated. It is a question of studying the valorization of the olive seed as follows: Lignin is extracted and acetylated. Then, a comparison of the natural versus chemically-altered structures of the lignin would take place. This comparison is based on several physico-chemical analyses to determine to which extent the Lignin's polymer value is suitable for the Synthesis of polyurethane foams [4-6].

Many studies at the laboratory level have focused on using lignins as macromonomers to prepare engineering plastics. In all cases, the $\mathrm{OH}$ functions (aliphatic and phenolic) were used to perform polycondensations with acid functions (formation of polyesters), isocyanates (formation of polyurethanes) or oxiranes (formation of polyethers) [7, 8].

The solubilization of lignin in various organic solvents generally used for NMR or size exclusion chromatography (SEC) is very limited and partial. It is often necessary to practice preceding acetylation. Acetylation of lignin with a pyridine / acetic anhydride mixture $(1 / 1, \mathrm{v} / \mathrm{v})$ is the most commonly used method. The purpose of this treatment is in particular to reduce the hydrophilicity of the lignin and to stabilize it dimensionally, by substituting the numerous hydroxyl groups present, with acetyl groups, which are larger and less polar [9].

All chemical modifications have been characterized at the molecular level, using Fourier transform infrared spectroscopy (FTIR), thermogravimetric analysis (TGA), differential scanning calorimetry analysis (DSC), NMR analysis C-13, 1H NMR and analysis sec.

\section{EXPERIMENTAL}

\subsection{Characterization of olive pomace}

The purpose of the thermogravimetric analysis is to follow the mass losses of a material subjected to a temperature variation. The thermal stability of lignins is evidenced by thermogravimetric analysis (TGA) and differential enthalpy analysis (DSC) [10, 11]. TGA and DSC avail for identifying Lignin's thermal stability under air needed for synthesizing several substances such as resin-like materials, glues or foam. In this case, TGA and DSC account for the type of inter-unit links and their percentage of chemical compositions $[12,13]$.

\subsection{Materials}

Exhausted olive pomace is naturally dried in the sun to a moisture content of $10 \%$ or less. Then, these samples are 
milled by a ball mill in order to have homogeneous products. Using sieves of different mesh sizes, uniform particle size was achieved - the final particles had diameters between 1 and 1.6 $\mathrm{mm}$. This powder will be easily analyzed and handled.

The preparation of samples for various characterization tests played a very important role in this determination of the reliability of the biomass and their importance in the manufacture of the polyol.

\subsection{Extraction of lignin by the organosolv method}

Initially, $100 \mathrm{~g}$ (oven-dry matter, dry matter content greater than $95 \%$ ) of spent olive-pomace was treated with ethanol/water $(65 / 35 \mathrm{v} / \mathrm{v})$ and sulfuric acid $(4 \mathrm{~N})(1,5) \%-\mathrm{w})$ used as a catalyst. The solid-liquid ratio applied was 1: 10. The mixture was heated in a 2-liter Parr reactor equipped with a Parr 4842 (Parr Instrument company) temperature controller to $170^{\circ} \mathrm{C}$ with stirring. The temperature was maintained 60 min before cooling and opening the reactor. Once the reaction mixture was at room temperature, it was filtered using a 12-15 $\mu \mathrm{m}$ cut-off-point filter paper, then washed with aqueous ethanol $(65 / 35 \mathrm{v} / \mathrm{v})$ at $60^{\circ} \mathrm{C}$. The filters containing the solid residue - called dough - were first dried at $40^{\circ} \mathrm{C}$ for $72 \mathrm{~h}$, then at $105^{\circ} \mathrm{C}$. Later, they were weighed in order to determine the mass of the solid residue. After combining the washes, 3 volumes of water were added to precipitate organosolv ethanol Lignin (EOL). At a final step, EOL was collected by centrifugation and dried in an oven at $105^{\circ} \mathrm{C}$ for $24 \mathrm{~h}$.

\subsection{Physico-chemical analyzes}

\subsubsection{Acetylation of lignin}

According to the method of Lin and Dence [14, 15], the lignin sample (about $100 \mathrm{mg}$ ) was acetylated with 1-2 $\mathrm{ml}$ of acetic anhydride: pyridine (1: $1, \mathrm{~V} / \mathrm{V})$ mixture, at room temperature overnight. A $50 \mathrm{ml}$ flask, $25 \mathrm{ml}$ of ethanol was added and evaporated $30 \mathrm{~min}$ later by rotary evaporator. The addition of $25 \mathrm{ml}$ of ethanol and the evaporations were repeated 5 to 10 times until all the acetic anhydride and pyridine were removed. The acetylated derivative was dissolved in 1-2 $\mathrm{ml}$ of chloroform and the solution was added drop wise in $100 \mathrm{ml}$ of diethyl ether with stirring. The precipitate, then, was centrifuged and washed with ether.

\subsubsection{Analysis of organosolv lignin sugars}

The monosaccharide contents of the soluble fraction were analyzed by HAPE-PAD (ICS-3000 Dionex) equipped with a DionexCarboPacTM PA-20 analytical column $(3 \times 150 \mathrm{~mm})$. The filtered samples $(20 \mu \mathrm{l})$ were eluted at $35^{\circ} \mathrm{C}$. and $0.4 \mathrm{ml} /$ min with the following composition: $99.2 \%$ pure water / 250 $\mathrm{mMNaOH} 0.8 \%$ : from 0 to $20 \mathrm{~min} ; 75 \%$ pure water $/ 250$ mMNaOH 20\% / NaOAc (1 M) - NaOH (20 mM) 5\% from 20 to $37 \mathrm{~min} ; 40 \%$ pure water / $250 \mathrm{mMNaOH} 20 \%$ / NaOAc (1 M) $-\mathrm{NaOH}$ (20 mM) 40\% from 37 to $41 \mathrm{~min}$. Each elution was followed by a wash and a subsequent equilibration time. External standards of sugar and uronic acids were used for calibration, mainly glucose, xylose, galactose, mannose, arabinose, (all provided by Sigma-Aldrich).

\subsubsection{Thermogravimetric analysis (TGA) and Differential scanning calorimetry (DSC)}

The thermogravimetric analysis was carried out in a TGA analyzer "Q500 V20.13 Build 39" with different heating rates $\left(5,10,15\right.$ and $\left.20^{\circ} \mathrm{C} . / \mathrm{min}\right)$ under an atmosphere of nitrogen $\left(\mathrm{N}_{2}\right)$ with a flow rate of $40 \mathrm{ml} / \mathrm{min}$ and at $20 \%$ oxygen on $35 \mathrm{mg}$ mass samples heated from 25 to $800^{\circ} \mathrm{C}$ in order to choose a heating rate for the thermal study of the mixtures. The samples were placed in uniform-sized particles in a 50 and $100 \mu \mathrm{L}$ platinum saucepan. Thermogravimetric analysis was conducted to evaluate the thermal behavior of simple lignin and acetyl lignin.

DSC analyses were carried out on a "DSC Q20 V24.10 Build 122" apparatus at a heating rate of $10^{\circ} \mathrm{C} . / \mathrm{min}$ under an inert nitrogen atmosphere of $50 \mathrm{ml} / \mathrm{min}$. Before the analysis, the samples were dried under vacuum at $40^{\circ} \mathrm{C}$. for one day. Then, about $5 \mathrm{mg}$ were weighed and encapsulated in an aluminum capsule. At the first sweep, the samples were heated from $-100^{\circ} \mathrm{C}$ to $200^{\circ} \mathrm{C}$, and then rapidly cooled with liquid nitrogen to eliminate the thermal history of the sample. The second sweep was then performed from $-100^{\circ} \mathrm{C}$ to $200^{\circ} \mathrm{C}$. The final thermogram resulted in the glass transition temperature Tg.

\subsubsection{Analysis FTIR}

FTIR spectra were collected on a BOMEN FTIR (MB 104 model) by preparing $\mathrm{KBr}$ pellets using a lignin concentration of $1 \%$ lignin. The spectra were recorded between 650 and 4000 $\mathrm{cm}^{-1}$ at a resolution of $4 \mathrm{~cm}^{-1}$. The recorded spectra were the average of 18 scans. In order not to contaminate the pellets with moisture, lignin (acetylated and lignin samples) and $\mathrm{KBr}$ were left for 24 hours at $40^{\circ} \mathrm{C}$ under reduced pressure prior to the pellets' preparation. It is important to note that the pellets were mastered at the same conditions for 12 hours before the FTIR analysis [16, 17].

\subsubsection{Analysis by size exclusion chromatography}

Size exclusion chromatography (SEC) was used to determine the average molecular weight $(\mathrm{Mw})$ and the number $(\mathrm{Mn})$ as well as the dispersity $(\mathrm{Mw} / \mathrm{Mn})$ of the lignin to be investigated.

The preparation of the lignin samples (acetylated or nonacetylated) to be injected was carried out accordingly: $0.04 \mathrm{~g}$ of lignin was dissolved in $15 \mathrm{ml}$ of solvent DMAC / LiCl $(0.5 \%)$ and stirred for at least 24 hours. After adding $5 \mathrm{ml}$ of solvent, a few $\mathrm{ml}$ of the homogenized solution was taken using a glass syringe and placed in the injection vial after filtering through a filter PTFE syringe with $0.45 \mu \mathrm{m}$ porosity.

Here are the conditions of the injection operating: The flow rate of the eluent $\mathrm{Q}$ was equal to $1 \mathrm{~mL} / \mathrm{min}$, the temperature of the column and the detector were 75 and $35^{\circ} \mathrm{C}$, respectively. The volume injected was $100 \mu \mathrm{L}$ for each test. The solvent used was DMAC / $\mathrm{LiCl}(0.5 \%$ by mass percentage $)$.

\section{RESULTS AND DISCUSSION}

\subsection{Physico-chemical analysis of simple and acetylated lignin}

The use of technical lignins in the manufacture of new products with high added value is hampered by several factors such as the complex, nonuniform and poorly-known structure of lignins. The first step in any lignin study must be the determination of the structural characteristics of the sample. As far as valorization is concerned, the characterization focuses on macromolecular and molecular properties.

There are several analytical methods described in the literature that characterize lignin [18]. 
Table 1. Acidity and alkalinity of lignin

\begin{tabular}{cccccc}
\hline Sample & Weight in grams & Initial pH & Volume $\mathbf{V}_{\mathbf{1}}$ in ml & Concentration of $\mathbf{~ N a O H ~ m o l ~ / ~ L ~}$ & Acidity mmol / g \\
\cline { 2 - 6 } & 1.5 & 3.50 & 0.35 & 0.1 & 2.33 \\
\hline
\end{tabular}

The initial $\mathrm{pH}$ and the acidity value are shown in Table 1 , which shows that after precipitation and washing of the lignin, the latter still contains a certain amount of acid impurities.

Table 2 reveals a few carboxylic functions and about 0.45 free phenolic groups per aromatic nucleus contained in lignin itself. This result remains acceptable and sufficiently high for derivatization by Oxypropylation.

Table 2. Analysis of total phenols and carbonyls by conductimetry

\begin{tabular}{ccc}
\hline & mmol/g lignin & mol/C9* \\
\hline Total Phenols & 2.18 & $0.45 \pm 0.01$ \\
Total carboxyl & 0.1 & $0.03 \pm 0.01$ \\
\hline Calculation based on a molar mass of C9 unit equal to $200 \mathrm{~g} / \mathrm{mol}$
\end{tabular}

\subsection{Analysis of sugars in lignin}

The neutral composition and content of sugar in lignin depend on several factors, such as its botanical origin, the process of transformation, and the extraction and purification stages used during its isolation. It is observed that the C5 sugars (pentoses) are dominant broadening (xylose), while the C6 sugars (glucose, galactose) are very little present. Consequently, the only sugars or hemicelluloses which remain on contact or associated with the residual lignin are of the arabinoxylan type, and note the total absence of mannans. The total and individual neutral sugar contents (arabinose, galactose, glucose, xylose, and mannose) presented in the lignin sample are summarized in Table 3.

\subsection{Analysis by Infra-Red Spectrometry (FTIR)}

Band assignments (FTIR) were based on data from the literature [19]. The main differences between the lignin samples are the signals due to acetylation (Figure 1), with the presence of the $\mathrm{C}=\mathrm{O}$ group around $1740 \mathrm{~cm}^{-1}$ in the case of acetylated lignin, and the absorption of the $\mathrm{OH}$ group at $3425 \mathrm{~cm}^{-1}$, less intense for acetylated lignin. The $\mathrm{CH}$ groups are observed at $2923 \mathrm{~cm}^{-1}$ for no-acetylated lignin and at $2939 \mathrm{~cm}^{-1}$ for acetylated lignin. It is possible to identify the characteristic bands of the syringyl $(\mathrm{S})$, guaiacyl $(\mathrm{G})$ and $\mathrm{p}$ hydroxyphenyl $(\mathrm{H})$ units as opposed to the different types of lignin (G, GS, HGS). The band at $1357 \mathrm{~cm}^{-1}$, specific to the cores $(\mathrm{S})$, the $830 \mathrm{~cm}^{-1}$ band bound to the $\mathrm{CH}$ groups in the $(\mathrm{S})$ and $(\mathrm{H})$ nuclei), along with the presence of an absorption band at $1220 \mathrm{~cm}^{-1}$ specific to the nucleus $(\mathrm{G})$ make it possible to consider that the skeleton of this lignin is of the HGS-type.

\subsection{Thermogravimetric analysis (TGA)}

The figure shows the thermogravimetric curves obtained for each lignin sample (acetylated and no-acetylated) under a nitrogen atmosphere (Figure 2).

Few differences appear overall in the thermal degradation profiles of the two lignin samples. The acetylation of lignin slightly increases its thermal stability. Whereas, at $600^{\circ} \mathrm{C}$, acetylated lignin tends to give a slightly lower residue than noacetylated lignin. The elimination of several impurities of lignin following acetylation can account for this difference. Respectively, the initial loss of mass at a temperature below $100^{\circ} \mathrm{C}$ seems to be higher in the case of no-acetylated lignin. The latter contains more residual moisture adsorbed on the free hydroxyl groups of lignin, which number is considerably reduced after acetylation (see IR spectra). Generally speaking, acetylation reduces the presence of residual hemicelluloses in lignin. Practically speaking, their presencein larger amounts in nonacetylated lignin - completely degraded at a temperature around $230^{\circ} \mathrm{C}$ (lower than for lignin) - is probably responsible for the lower decay rate, between 250 and $450^{\circ} \mathrm{C}$, and the mass of no-acetylated lignin. The Percentage of Chemical composition in dry Olive Pomaceis estimated at 22.8 Hemicellulose, 24.6 Cellulose, and 45.2 lignin.

Table 3. Residual sugar content in lignin

\begin{tabular}{cccccc}
\hline & Arabinose & Galactose & Glucose & Xylose & Mannose \\
\hline Sugars (\%, w / w) & 0.692 & 2.041 & 3.252 & 19.223 & ndt \\
Total sugars (\%, w/ w) & \multicolumn{7}{c}{25.208} \\
\hline \multicolumn{6}{c}{ ndt: not detected } \\
\hline
\end{tabular}

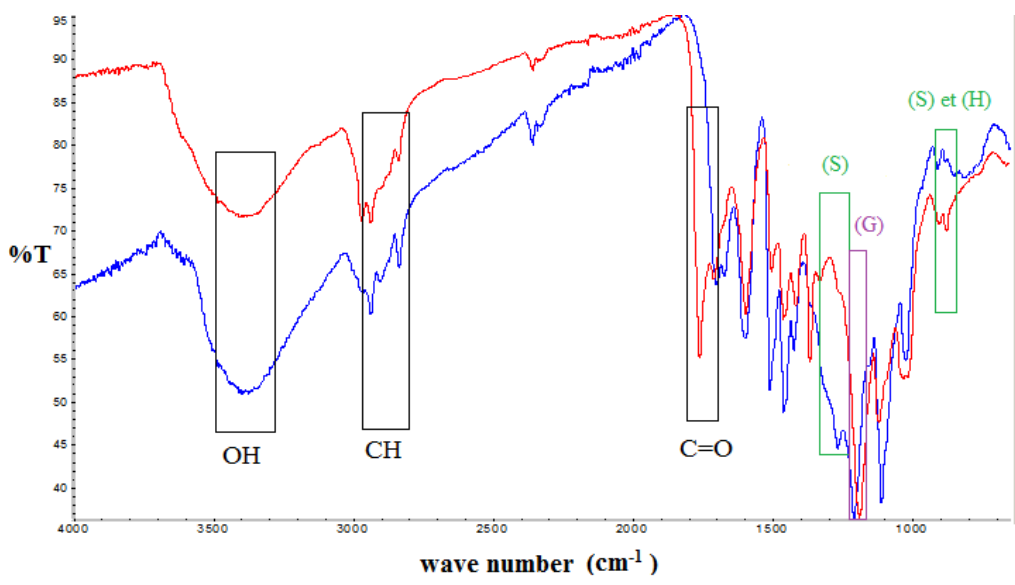

Figure 1. Comparison of FTIR spectra of no-acetylated lignin and acetylated lignin 


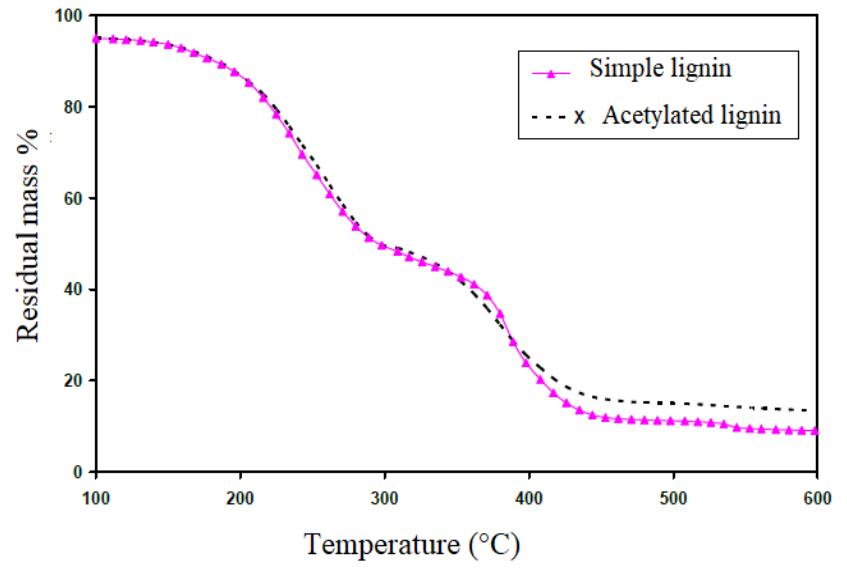

Figure 2. Thermogravimetric curves of simple lignine and acetylated lignin

\subsection{Differential scanning calorimetry (DSC)}

As far as the observation of the temperature domains registering physicochemical processes is concerned, it is safe to mention that DSC is more precise than TGA. To this end, the curves in Figure 3 record two processes:

-An endothermic process $(\phi<0)$ between 30 and $150^{\circ} \mathrm{C}$ corresponding to the evaporation of water bound to the solid matrix (after the initial nitrogen sweep phase, at room temperature, preceding the DSC analyzes, intended to evaporate water weakly bound to the matrix, as well as highly volatile organic compounds).

-An exothermic process $(\phi>0)$ that starts from $200^{\circ} \mathrm{C}$ for lignin, and about $250^{\circ} \mathrm{C}$ for acetylated lignin. This second process, which continues and increases with the rise of temperature, corresponds to the pyrolysis of the material, lignin, and residual polysaccharides possibly present. Another important observation is that the amplitude of the 1st process is registered lower for acetylated lignin ( $\phi$ and lower endothermic peak area). This implicates that the acetylated lignin is less hydrophilic. Furthermore, the pyrolysis of the acetylated lignin (2nd process) begins at a higher temperature. It is safe to note that the thermal stability of the latter is improved. This difference in thermal behavior after acetylation is consistent with the general results of the literature: The decrease in polarity of lignin, due to the disappearance of free $\mathrm{OH}$ functions, generally increases the hydrophobicity and thermal stability of lignin.

\subsection{RMN analysis C-13 and $1 \mathrm{H}-\mathrm{NMR}$}

The samples (simple lignin and acetylated) in powder form are placed in a boron nitride sample holder $3 \mathrm{~mm}$ in diameter and $6 \mathrm{~mm}$ high. A contact time of 0.8 seconds and a delay of 20 seconds between sequences and rotation between 5 and 6 $\mathrm{kHz}$ were used. An average of 1000 contacts was required to have an interpretable spectrum (Figure 4).

In the 13C-NMR spectrum of the original lignin (Figure 5), the signal observed at $168.9 \mathrm{ppm}$ is found to interfere with the determination of the phenolic hydroxyl content. The integration of the individual contributions using the acetylation procedure shows carboxyl and carbonyl between 160 and 200 ppm, methoxyl from 54 to $58 \mathrm{ppm}, \mathrm{C}$ from $\beta$-O4 between 81 and 88 ppm, and aliphatic $\mathrm{CH}$ between 48 and $100 \mathrm{ppm}$. On the acetylated lignin spectrum, the hydroxyl content is calculated by taking the following integration limits: primary alcohols between 169.4 and 170.4 ppm, secondary alcohols from 168.5 to $169.4 \mathrm{ppm}$, and phenolic hydroxyls from 165.8 to $168.5 \mathrm{pp}$. The values obtained from the integrals of the signals are normalized with respect to the integral of the C9 units (phenylpropane).

In order to compare the results obtained from different techniques, the previous established molecular weights of $\mathrm{C} 9$ lignin base units are used in the calculation of total hydroxyl content in mmol / g, from 13-C NMR (obtained as $\mathrm{OH} / \mathrm{C} 9$ unit). For a better study of lignin by 13-C NMR, the acetylation of lignin is carried out. This analysis makes it possible to derive the alcohols from the aliphatic chain as well as the free phenolic groups. Indeed, the hydroxyl groups are replaced by acetyl groups. Thus, the signals obtained for carboxylates from primary alcohols, secondary alcohols, and phenolic alcohols are identified as shown in the spectrum of Figure 5.
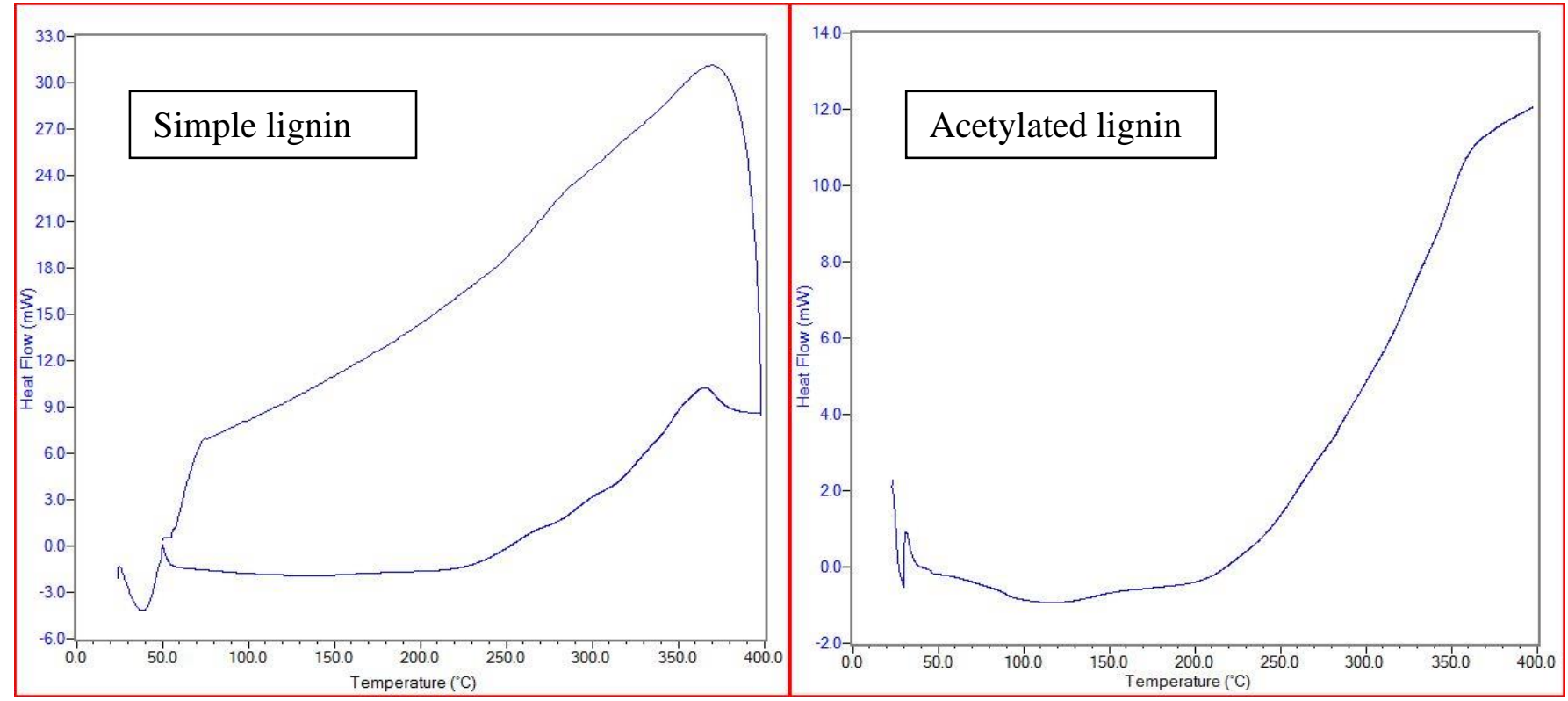

Figure 3. Differential scanning calorimetry analysis of simple lignin and acetylated lignin 


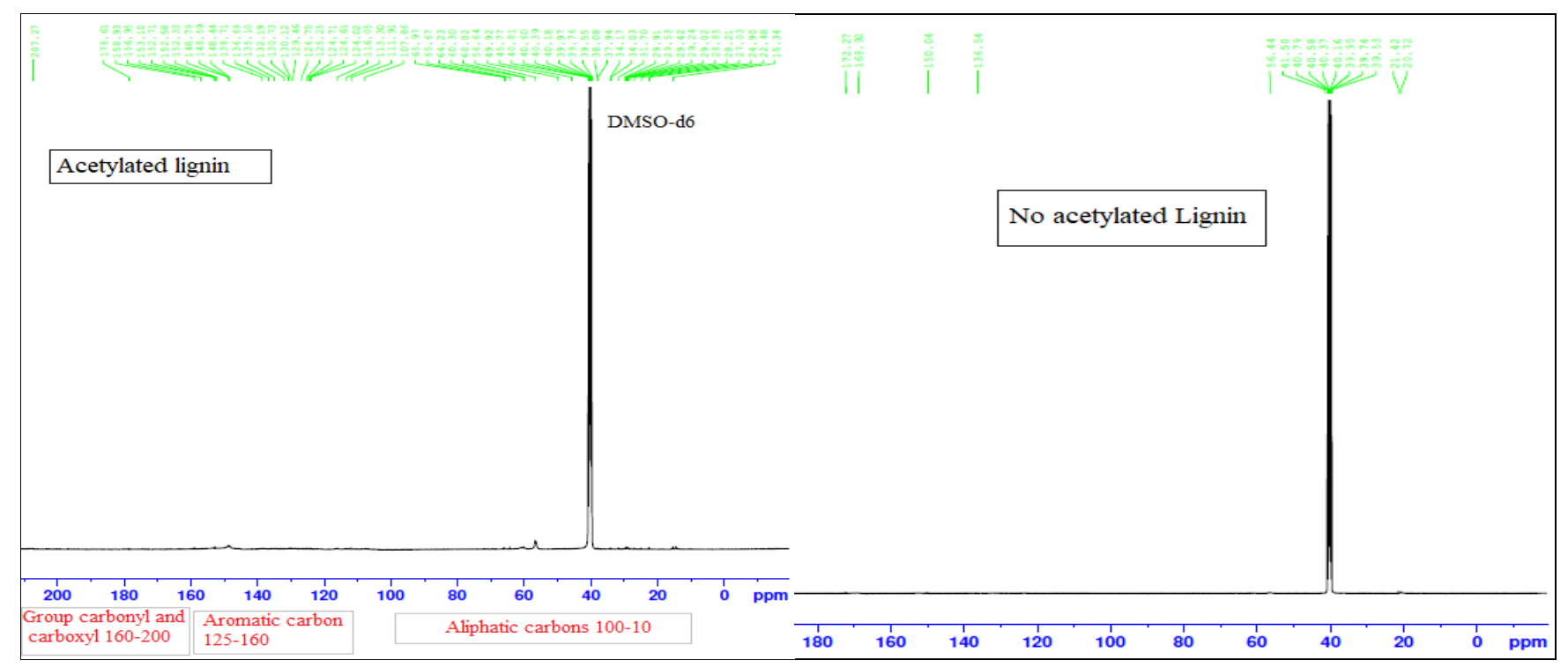

Figure 4. 13C-NMR spectrum of acetylated lignin and lignin original

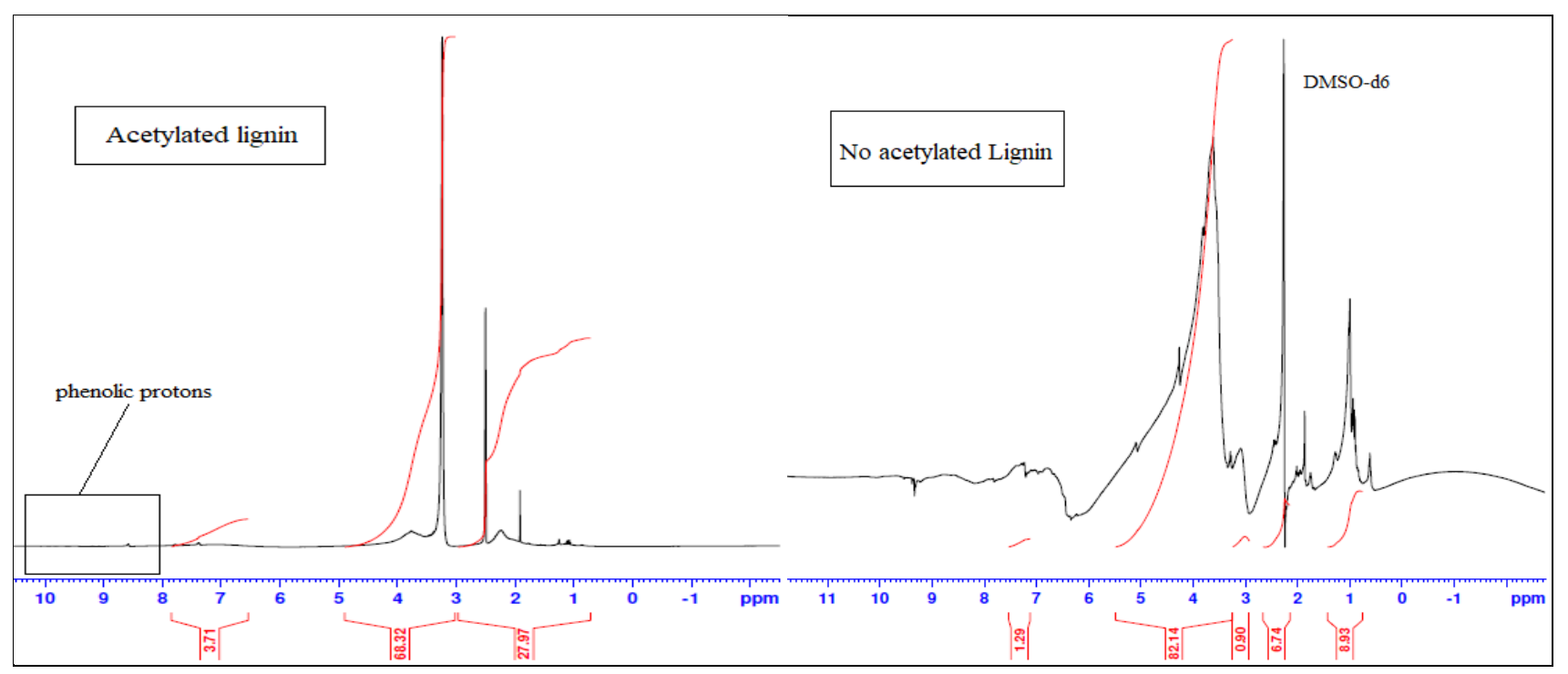

Figure 5. 1H-NMR spectrum of acetylated lignin and lignin original

Table 4. Molar mass distribution of no-acetylated and acetylated lignin

\begin{tabular}{cccc}
\hline Lignin organosolv & Average mass by weight (Mw) & Average mass in number (Mn) & Dispersity (Mw / Mn) \\
\hline Acetylated lignin & 5378 & 257 & 20.92 \\
Simple lignin & 1092 & 108 & 10.11 \\
\hline
\end{tabular}

The results of the molar mass distribution are shown in the table.

Table 4 reveals that acetylation increases the weight and number of molar masses by 25 and $50 \%$, respectively. Acetylation causes a greater effect on short chains that are probably richer in free phenolic groups.

By the same token, the polydispersity values are very high $(20>>1)$, while the lignins may consist of both galactoglucomannan-lignin and glucomannan-lignin complexes. The presence of both complexes could partially explain the high polydispersity of the compounds, considering they would have two types of molecules of different sizes [20].

\section{CONCLUSIONS}

The lignin samples (acetylated and no-acetylated) were characterized through several analytical techniques and methods, having as their point of view their valorization as polyols in the polyurethane synthesis. Lignin samples had properties inherent to their origin, as well as corresponding extraction and precipitation processes. The other characteristics, namely thermal, are the ones generally expected of alkaline lignin.

It seems lignin has the most peculiar characteristics. Hydroxyphenyl-guaiacyl-syringyl (HGS)-type contains $25.2 \%$ sugar and $2.33 \mathrm{mmol} / \mathrm{g}$ of acid. The phenolic hydroxyl content shows comparable results to 13C-NMR along with the advantage of allowing quantification by the discrimination of the aromatic hydroxyl groups attached to the syringyl, guaiacile, and p-hydroxyphenyl units.

Overall, this lignin showcases adequate features, mainly a sufficient quantity of hydroxyl functional groups. Consequently, it can safely serve as a base polymer for the 
production of oxypropylated derivatives for the formation of polyurethane foams.

\section{REFERENCES}

[1] Tunisie en chiffres/Statistiques Tunisie. (2018). ISSN: 0330-6739.

http://www.ins.tn/sites/default/files/publication/pdf/tec2019-v11-web2.pdf, accessed on 20 August 2020.

[2] Chebil, A. (2011). Strategy for the development of Tunisian exports of processed fruits and vegetables. Working document of the Ministry of Trade and tourism, Tunisia.

[3] Grondahl, M., Teleman, A., Gatenholm, P. (2003). Effect of acetylation on the material properties of glucuronoxylan from aspen wood. Carbohydrate Polymers, 52(4): 359-366. https://doi.org/10.1016/S0144-8617(03)00014-6

[4] Perin-Levasseur, Z., Savulescu, L., Benali, M. (2011). Lignin production path assessment: Energy, water, and chemical integration perspective. Journal of Science \& Technology for Forest Products and Processes, 1(3): 2530.

[5] Strassberger, Z., Tanase, S., Rothenberg, G. (2014) The pros and cons of lignin valorisation in an integrated biorefinery. RSC Advances, 4: 25310-25318.

[6] Adler, E. (1977). Lignin chemistry-past, present and future. Wood Science and Technology, 11: 169-218. https://doi.org/10.1007/BF00365615

[7] Mekki, H., Anderson, M., Ben Zina, M., Ammar, E. (2008). Valorization of olive mill wastewater by its incorporation in building bricks. Journal of Hazardous Materials, $\quad$ 158(2-3): 308-315. https://doi.org/10.1016/j.jhazmat.2008.01.104

[8] El Mansouri, N.E., Salvadó, J. (2006). Structural characterization of technical lignins for the production of adhesives: Application to lignosulfonate, kraft, sodaanthraquinone, organosolv and ethanol process lignins. Industrial Crops and Products, 24(1): 8-16. https://doi.org/10.1016/j.indcrop.2005.10.002

[9] Tawarah, K.M., Rababah, R.A. (2003). Characterization of some Jordanian crude and exhausted olive pomace samples. Green and Sustainable Chemistry, 3(4): 146162. https://doi.org/10.4236/gsc.2013.34018

[10] Yorulmaz, S.Y., Atimtay, A.T. (2009). Investigation of combustion kinetics of treated and untreated waste wood samples with thermogravimetric analysis. Fuel Processing Technology, 90(7-8): 939-946. https://doi.org/10.1016/j.fuproc.2009.02.010

[11] Neto, C.G.T., Giacometti, J.A., Job, A.E., Ferreira, F.C., Fonseca, J.L.C., Pereira, M.R. (2005). Thermal analysis of chitosan based networks. Carbohydrate Polymers, 62(2): 97-103. https://doi.org/10.1016/j.carbpol.2005.02.022

[12] Kittur, F.S., Prashanth, K.V.H., UdayaSankar, K., Tharanathan, R.N. (2002). Characterization of chitin and their carboxymethyl derivatives by differential scanning calorimetry. Carbohydrate Polymers, 49(2): 185-193. https://doi.org/10.1016/S0144-8617(01)00320-4

[13] Gandini, A., Belgacem, M.N. (2008). Lignin as components of macromolecular materials. In: Belgacem, M.N., Gandini, A. (Eds.), Monomers, Polymers and Composites from Renewable Resources. Elsevier, Amsterdam, 243-271.

[14] Garcia-Ibaňes, P., Sáchez, M., Cabanillas, A. (2006). Thermogravimetric analysis of olive oil residue in air atmosphere. Fuel Processing Technology, 87(2): 103107. https://doi.org/10.1016/j.fuproc.2005.08.005

[15] Demirbas, A. (2004). Effects of temperature and particle size on bio-char yield from pyrolysis of agricultural residues. Journal of Analytical and Applied Pyrolysis, 72(2): https://doi.org/10.1016/j.jaap.2004.07.003

[16] Dence, C.W. (1992). Determination of Carboxylic Groups. In: Lin, E.S., Dence, C.W. (Eds.), Methods in Lignin Chemistry. Springer-Verlag, Berlin-Heidelberg, 3-6.

[17] Sun, R.C., Xue, B.L., Wen, J.L. (2015). Ethanol organosolv lignin as a reactive filler for acrylamidebased hydrogels. Journal of Applied Polymer Science, 132(40). https://doi.org/10.1002/APP.42638

[18] Vishtal, A., Kraslawski, A. (2011). Chanllenges in industrial applications of technical lignins. Bioresources, 6(3): 3547-3568.

[19] Cateto, C.A., Barreiro, M.F., Rodrigues, A.E., BrochierSalon, M.C., Thielemans, W., Belgacem, M.N. (2008). Lignins as macromonomers for polyurethane synthesis: A comparative study on hydroxyl group. determination. Journal of Applied Polymer Science, 109(5): 3008-3017. https://doi.org/10.1002/app.28393

[20] Nadji, H., Bruzzèse, C., Belgacem, M.N., Benaboura, A., Gandini, A. (2005). Oxypropylation of lignins and preparation of rigid polyurethane foams from the ensuing polyols. Macromolecular Materials and Engineering, 290(10): https://doi.org/10.1002/mame.200500200 